Article

\title{
Design of Programmable LED Controller with a Variable Current Source for 3D Image Display
}

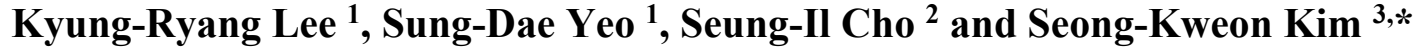

1 Graduate School of NID Fusion Technology, Seoul National University of Science \& Technology, 232 Gongneung-ro, Nowon-gu, Seoul, Korea; E-Mails: corenc@seoultech.ac.kr (K.-R.L.); ysd1009@seoultech.ac.kr (S.-D.Y.)

2 Innovation Center for Organic Electronics, Yamagata University, 1-808-48 Arcadia, Yonezawa, Yamagata 992-0119, Japan; E-Mail: cho_si@yz.yamagata-u.ac.jp

3 Department of Electronic and IT Media Engineering, Seoul National University of Science \& Technology, 232 Gongneung-ro, Nowon-gu, Seoul, Korea

* Author to whom correspondence should be addressed; E-Mail: kim12632@seoultech.ac.kr; Tel.: +82-02-970-6432; Fax: +82-02-974-6123.

External Editors: Jason C. Hung, Shu-Ching Chen and Young-Sik Jeong Received: 14 August 2014; in revised form: 13 October 2014 / Accepted: 5 November 2014 / Published: 3 December 2014

\begin{abstract}
Conventional fluorescent light sources, as well as incandescent light sources are gradually being replaced by Light Emitting Diodes (LEDs) for reducing power consumption in the image display area for multimedia application. An LED light source requires a controller with a low-power operation. In this paper, a low-power technique using adiabatic operation is applied for the implementation of LED controller with a stable constant-current, a low-power and low-heat function. From the simulation result, the power consumption of the proposed LED controller using adiabatic operation was reduced to about $87 \%$ in comparison with conventional operation with a constant $V_{D D}$. The proposed circuit is expected to be an alternative LED controller which is sensitive to external conditions such as heat.
\end{abstract}

Keywords: light emitting diode (LED); adiabatic; constant current; low power 


\section{Introduction}

There have been a number of methodological attempts for energy conservation in image display area for multimedia application. In lighting and image display area, the light emitting diode (LED) system has been studied. Also, conventional fluorescent light sources, as well as incandescent light sources are gradually being replaced by LEDs. An LED light source is often used with a controller for low-power operation. Increasing the LED channel, an LED controller has been required to be operated with a low-power design. In particular, the low power design of a display controller becomes an essential block in a study of 3D image display [1].

In this paper, we introduce the idea of reducing the power consumption of an LED lighting controller through low-power adiabatic methodology. So far, various attempts have been made in the limited condition of a constant power supply voltage without any control [2]. However, there has not been a dramatic reduction of power consumption. The general energy consumption of a Complementary Metal Oxide Semiconductor (CMOS) circuit is caused by the channel ON resistance in switching time of transistor.

A typical LED driving system generates a large driving current with analog or pulse width modulation (PWM) dimming to represent the brightness of an LED device [3]. Because a large current flows through channel resistance RoN of the MOS transistor, large power consumption occurs. The power consumption of ON resistance degraded the performance of the LED device. An LED device with temperature-sensitive characteristics can make the designed output characteristics worse. In many case, this effect has caused the destruction of LED driving system.

A low power circuit design method using adiabatic operation is to transmit adiabatic signal of ON resistance [4]. When this method for designing a low power LED application is applied, the power consumption of a large current driving LED system can be reduced. Instability resulting from overheating of an LED system can be overcome. Therefore, in this paper, an adiabatic method for lowpower operation is utilized for implementing an LED controller.

\section{Adiabatic Operation}

Figure 1 shows the voltage variation of source $\left(\mathrm{V}_{\mathrm{DD}}\right)$, capacitance $\left(\mathrm{V}_{\mathrm{C}}\right)$ and resistance $\left(\mathrm{V}_{\mathrm{RON}}\right)$ respectively, in a conventional MOS pull-up network. A transistor is assumed to be a constant current source that can move the energy of $\mathrm{CV}^{2}$ on the period of time. $\mathrm{V}_{\mathrm{R}}$ represents the variation of the transistor channel voltage supplied from the drain. $V_{C}$ represents the load voltage variation from the charged voltage. Rapid and large voltage variation at resistance cause a circuit to experience a large power dissipation due to the thermal loss [5].

Figure 2 shows the voltage variation of source $\left(\mathrm{V}_{\text {TRI }}\right)$, capacitance $\left(\mathrm{V}_{\mathrm{C}}{ }^{\prime}\right)$ and resistance $\left(\mathrm{V}_{\mathrm{RON}}{ }^{\prime}\right)$ respectively, in an adiabatic MOS pull-up network. When a voltage source with a long time constant of $\tau$, such as ramped voltage $V_{I}$, was applied to transistor through drain, there was a small voltage variation that shows a little power consumption in the transistor. Theoretically, if the time constant approaches infinity, the energy consumption of resistance converges to zero [6]. 
Figure 1. MOS pull-up network VRON energy consumption.

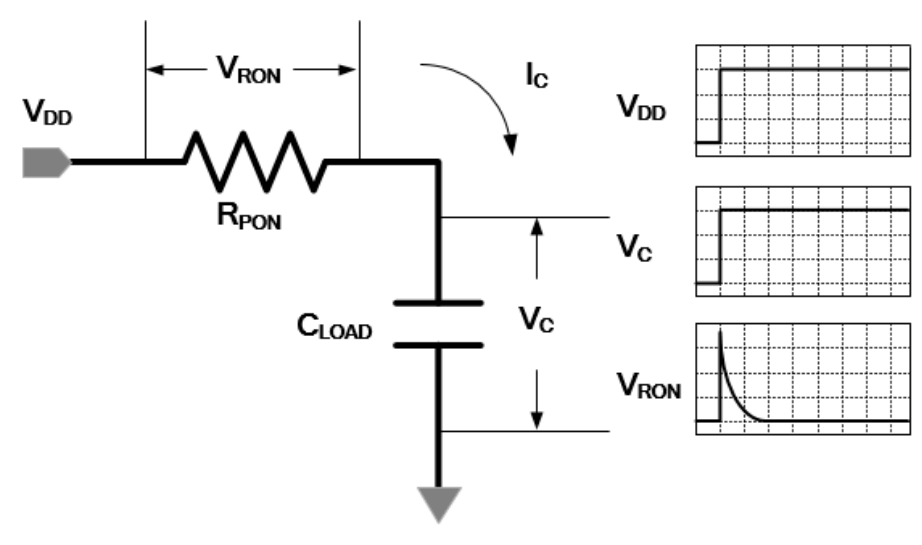

Figure 2. Adiabatic pull-up network VRON' energy consumption.

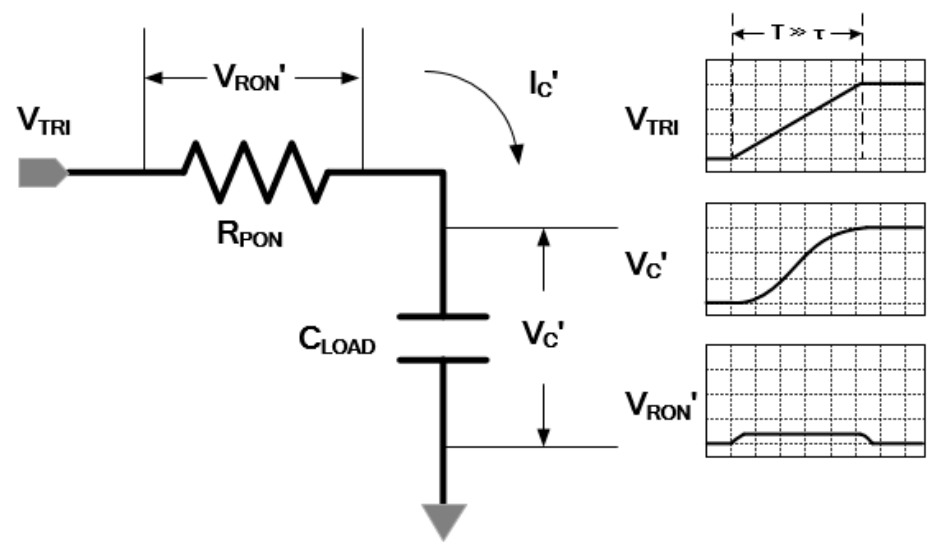

$V_{\text {RON }}$ is the change of voltage on the channel resistance RPON from step voltage source VDD at pull-up network in Figure $1 V_{\text {RON }}$ can be expressed as:

$$
\mathrm{V}_{\mathrm{DD}}=\mathrm{R}_{\mathrm{PON}} \mathrm{I}_{\mathrm{C}}(\mathrm{t})+\mathrm{V}_{\mathrm{C}}(\mathrm{t})
$$

where $I_{C}$ is the displacement current across the load capacitance voltage $\mathrm{V}_{\mathrm{C}}$. Ic can be obtained from:

$$
I_{C}(t)=C \frac{d V_{C}(t)}{d t}
$$

Load capacitance voltage, $V_{C}$ and pull-up path current, $I_{C}(t)$ is calculated as:

$$
V_{C}(t)=V_{D D}\left(1-e^{-\frac{t}{C R_{P O N}}}\right), I_{C}(t)=\frac{V_{D D}}{R_{P O N}} e^{-\frac{t}{C R_{P O N}}}
$$

Power dissipation at load capacitance can be calculated as $\mathrm{V}_{\mathrm{C}}(\mathrm{t}) \times \mathrm{IC}_{\mathrm{C}}(\mathrm{t})$. MOS Ron power dissipation can be expressed as:

$$
\mathrm{P}_{\text {LOADCAP }}=\mathrm{V}_{\mathrm{C}}(\mathrm{t}) \cdot \mathrm{I}_{\mathrm{C}}(\mathrm{t})=\frac{\mathrm{V}_{\text {DD }}^{2}}{\mathrm{R}_{\text {PON }}} \mathrm{e}^{-\frac{\mathrm{t}}{\mathrm{C} \mathrm{C}_{\text {PON }}}}\left(1-\mathrm{e}^{-\frac{\mathrm{t}}{\mathrm{CR_{ \text {PON } }}}}\right), \mathrm{P}_{\mathrm{R}_{\text {PON }}}=\frac{\mathrm{V}_{\text {DD }}^{2}}{\mathrm{R}_{\text {PON }}} \mathrm{e}^{-\frac{2 \mathrm{t}}{\mathrm{CR_{ \text {PON } }}}}
$$

Therefore, total energy dissipation of pull-up path can be calculated as:

$$
\mathrm{w}_{\mathrm{C}}(\mathrm{t})+\mathrm{w}_{\mathrm{R}_{\mathrm{ON}}}(\mathrm{t})=\frac{1}{2} \mathrm{CV}_{\mathrm{DD}}^{2}+\frac{1}{2} \mathrm{CV}_{\mathrm{DD}}^{2}=\mathrm{CV}_{\mathrm{DD}}^{2}
$$

where total energy dissipation is equal to supply energy. 
VRON' is the change of voltage on the channel resistance RPON' from the ramped voltage source VTRI, with the delay time of $\tau$, at pull-up network in Figure 2. IC' is the displacement current from load capacitance voltage $\mathrm{V}_{\mathrm{C}^{\prime}}$. $\mathrm{V}_{\mathrm{C}}$ and $\mathrm{IC}_{\mathrm{C}}^{\prime}$ are calculated as:

$$
\begin{aligned}
& \mathrm{V}_{\mathrm{C}}(\mathrm{t})^{\prime}=\frac{\mathrm{V}_{\text {TRI }}}{\tau}\left[\mathrm{t}-\mathrm{CR}_{\text {PON }}{ }^{\prime}\left(1-\mathrm{e}^{-\frac{\mathrm{t}}{\mathrm{CR_{ \text {PON } }}}}\right)-\left[(\mathrm{t}-\tau)-\mathrm{CR}_{\text {PON }}{ }^{\prime}\left(1-\mathrm{e}^{-\frac{\mathrm{t}}{\mathrm{CR} \mathrm{RON}^{\prime}}}\right)\right] \mathrm{u}(\mathrm{t}-\tau)\right], \\
& \mathrm{I}_{\mathrm{C}}(\mathrm{t})^{\prime}=\frac{\mathrm{CV}_{\text {TRI }}}{\tau}\left[\left(1-\mathrm{e}^{-\frac{\tau}{\mathrm{C \textrm {C } _ { \text { por } }}}}\right)-\left(1-\mathrm{e}^{-\frac{\tau}{\mathrm{CR} \text { pow' }}}\right)(\mathrm{t}-\tau)\right]
\end{aligned}
$$

Therefore, the total energy consumption of adiabatic pull-up path can be expressed as:

$$
\mathrm{w}_{\mathrm{C}}(\mathrm{t})+\mathrm{w}_{\mathrm{R}_{\mathrm{ON}}}(\mathrm{t})=\frac{\mathrm{CV}_{\mathrm{TRI}}^{2}}{2}+\frac{\mathrm{R}_{\mathrm{P}}{ }^{\prime} \mathrm{C}^{2} \mathrm{~V}_{\mathrm{TRI}}^{2}}{\tau}\left[1-\frac{\mathrm{CR}_{\mathrm{PON}}{ }^{\prime}}{\tau}\left(1-\mathrm{e}^{-\frac{\tau}{\mathrm{C} \mathrm{CPON}^{\prime}}}\right)\right]=\mathrm{CV}_{\mathrm{TRI}}^{2}
$$

where total energy dissipation is equals to supply energy.

When ramped voltage source $\mathrm{V}_{\text {TRI }}$ with delay time of $\tau$, is supplied to adiabatic circuit design, energy consumption, $\mathrm{CV}^{2} / 2$ in conventional MOS RON resistance can be reduced to an amount that can be expressed as $\mathrm{CV}_{\mathrm{TRI}^{2}}$.

\section{Current LED Controller Using Adiabatic Operation}

Figure 3 shows the proposed design of the adiabatic digital current LED controller. The current channel columns of the MOSFET are constituted according to the power of two for the LED drive current with an exponential function. Linearity of the output current can be achieved by the ON/OFF switch. The amount of the LED dimming can be determined in each binary switch current. Output current is determined with the LED specification. The current rating of the proposed design is determined with $25 \mathrm{~mA}$ by typical intermediate current of LED.

Figure 3. Current Light Emitting Diode (LED) control design circuit using adiabatic operation.

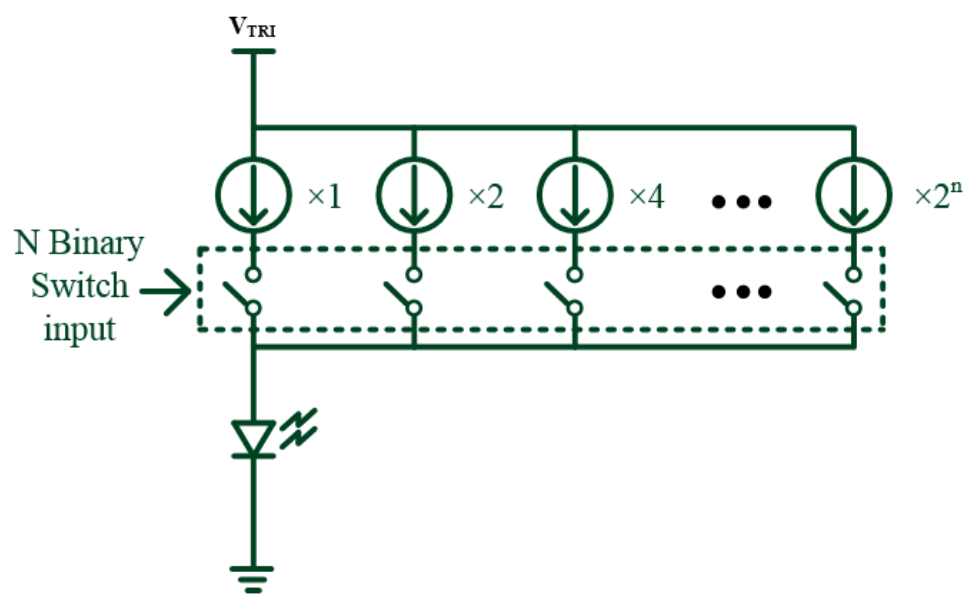

Figure 4 shows the basic circuit of the proposed LED controller with adiabatic operation. The basic circuit was designed with a high output impedance of two-stage PCH. It is a feature of LED that the current is determined with an exponential voltage change. A utilized MOS transistor is saturated when the switch is high. The LED's utilized load is an equivalent circuit which is constituted with $100 \Omega$ resistance in the condition of a typical voltage of $2.5 \mathrm{~V}$ with a current of $25 \mathrm{~mA}$. The zener diode that is used for safe operation of the LED is represented with $1 \mathrm{nF}$ capacitance. 
Figure 4. Basic circuit of LED controller with adiabatic operation.
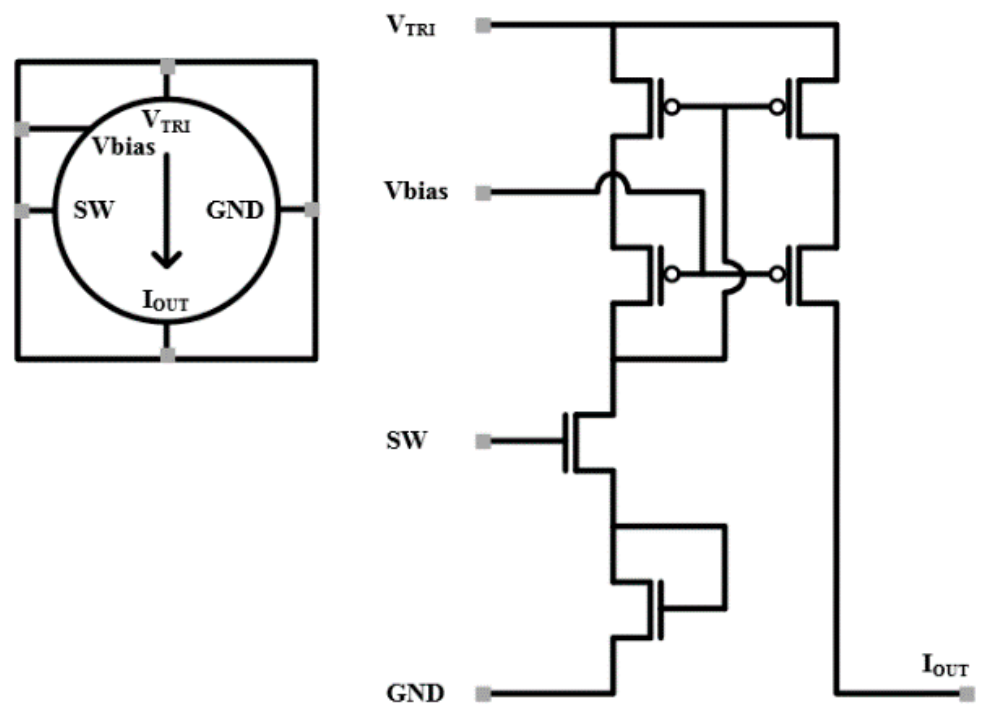

Figure 5 shows the I/V characteristic with $0.35 \mu \mathrm{m}$ CMOS process technology by the Dong-Bu company.

Figure 5. I/V characteristic of the used MOS transistor.

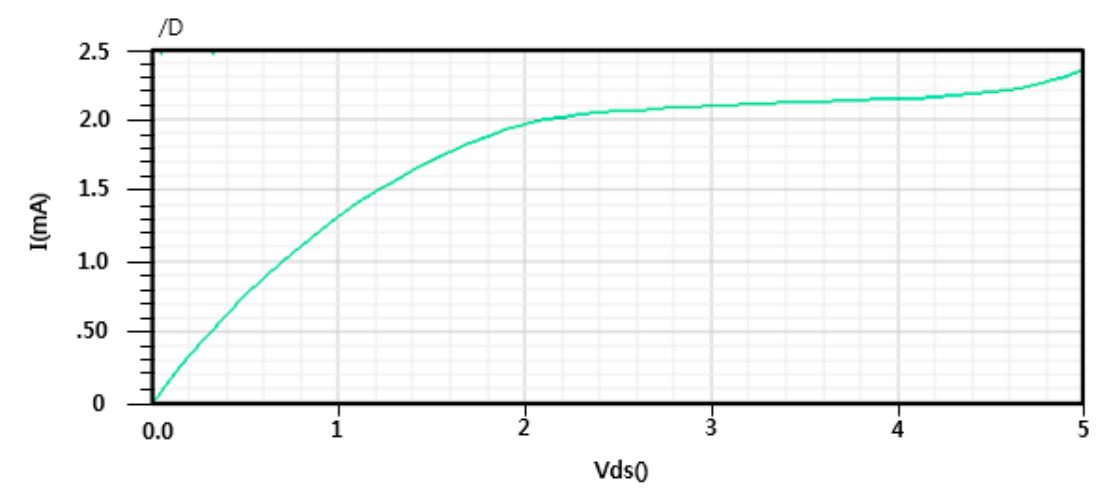

Figure 6 shows the characteristics of $\mathrm{VDS}_{\mathrm{DS}} / \mathrm{IDS}=\mathrm{RoN}$. The time constant is $2^{-15} \mathrm{~s}$ in the condition of a constant $\mathrm{VDS}_{\mathrm{DS}}=5 \mathrm{~V}$, and the resistance of the MOS transistor is $2 \mathrm{~m} \Omega$. Load capacitance is $1 \mathrm{pF}$. Ramped $\mathrm{V}_{\mathrm{DS}}$ is characterized with a cycle with $0.5-6 \mathrm{~s}$ and the time constant can be expressed as $0.25-6 \mathrm{~s}$ as a maximum value. If $V_{D S}$ is not a peak value, $V_{D S}$ is always smaller than $5 \mathrm{~V}$; therefore, the time constant of the ramped voltage of $V_{D S}$ is calculated as $0.25^{-6} \mathrm{~s} \times 63 \%=0.15^{-6} \mathrm{~s}$.

Figure 6. Ron characteristic of the used MOS transistor.

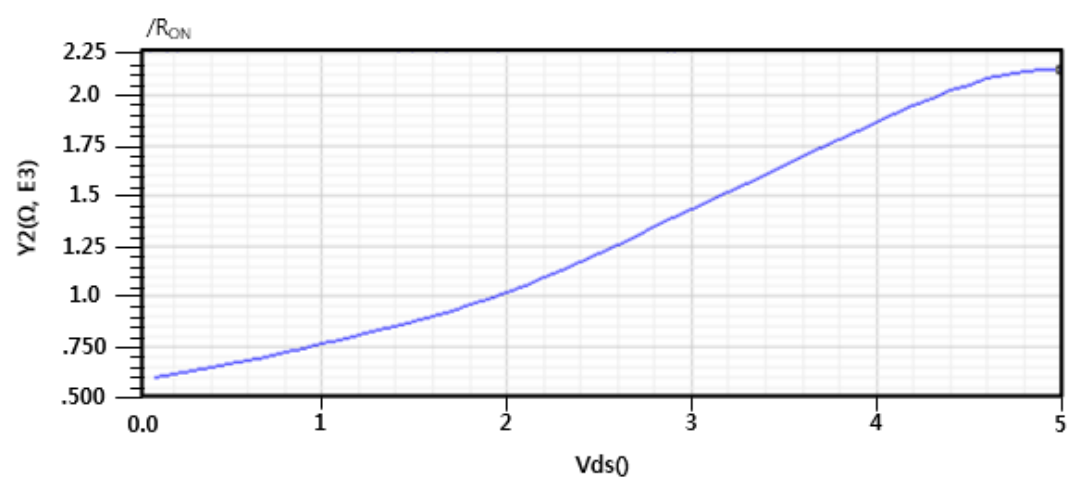


Figure 7 shows the power dissipation in comparison with the constant VDD and ramped VDD operation of the designed circuit. The energy dissipation of the constant $V_{D D}$ operation in the period time of $0 \sim 100 \mu \mathrm{s}$ is $16 \mu \mathrm{W}$. Energy dissipation of the ramped $\mathrm{VDD}_{\mathrm{DD}}$ operation is $3 \mu \mathrm{W}$. The basic circuit with adiabatic operation in comparison with conventional operation shows an energy reduction of $82 \%$.

Figure 7. Power consumption comparison between constant $V_{D D}$ operation and ramped $V_{D D}$.

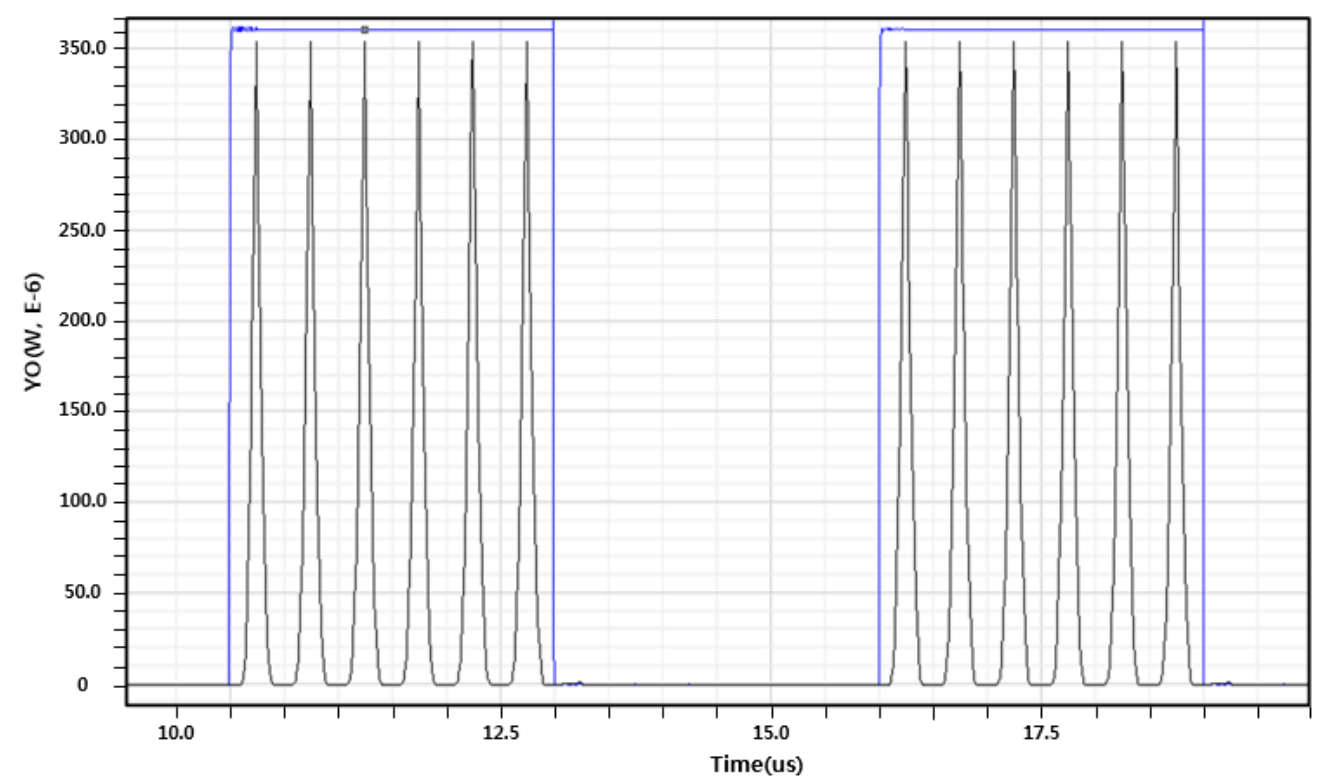

Figure 8 shows the LED controller structure that has 6-channel switches to the 63 basic circuits. Output current, LEDCHIouT shows the linear characteristic.

Figure 8. 6-bits current LED controller structure using adiabatic operation.

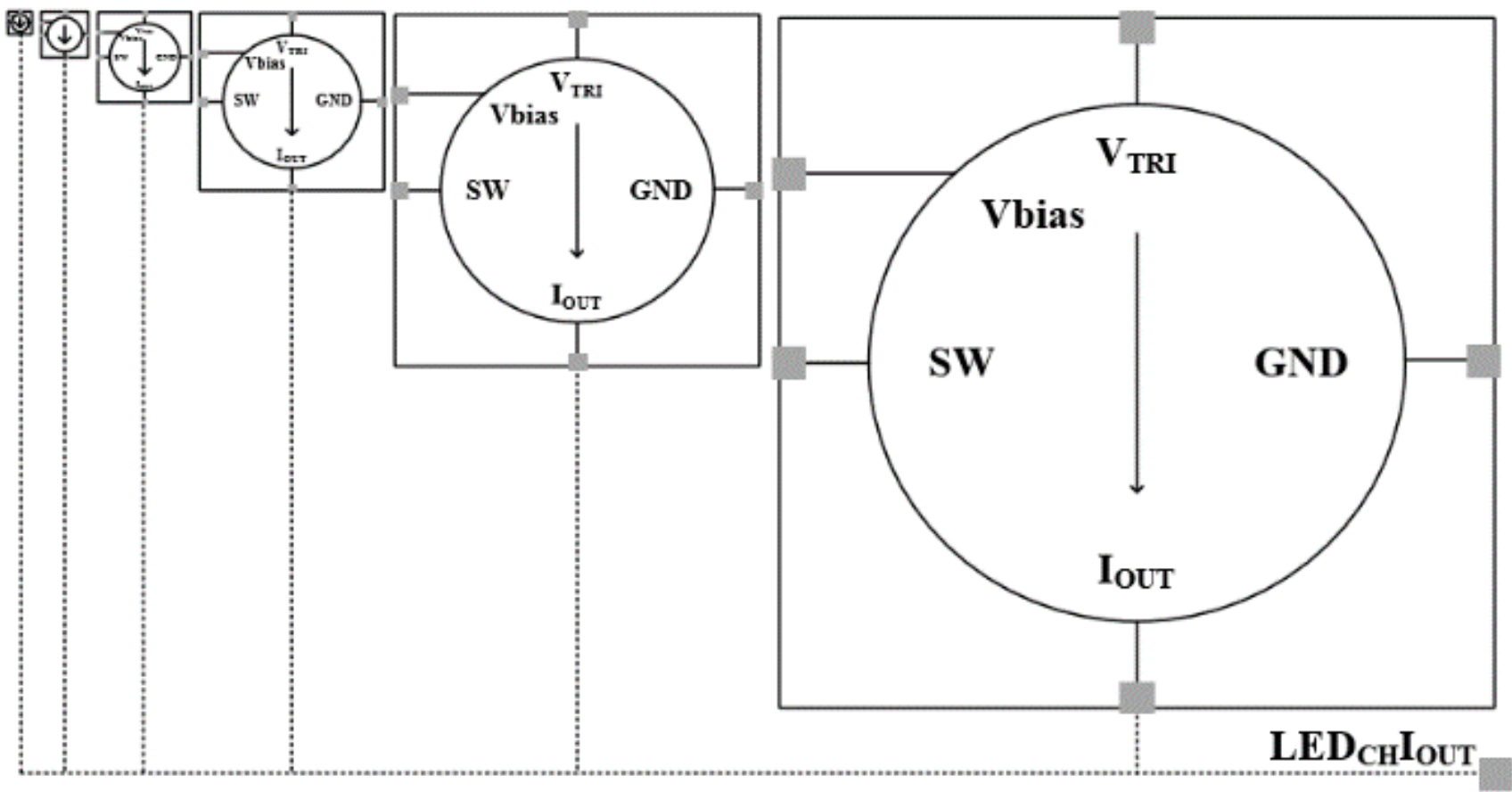


Figure 9 shows the photograph of the designed LED controller. The LED 4-channels with adiabatic operation were implemented with $0.35 \mu \mathrm{m}$ CMOS process technology by the Dong-Bu company and the size was $1400 \mu \mathrm{m} \times 700 \mu \mathrm{m}$.

Figure 9. Photograph of LED controller.

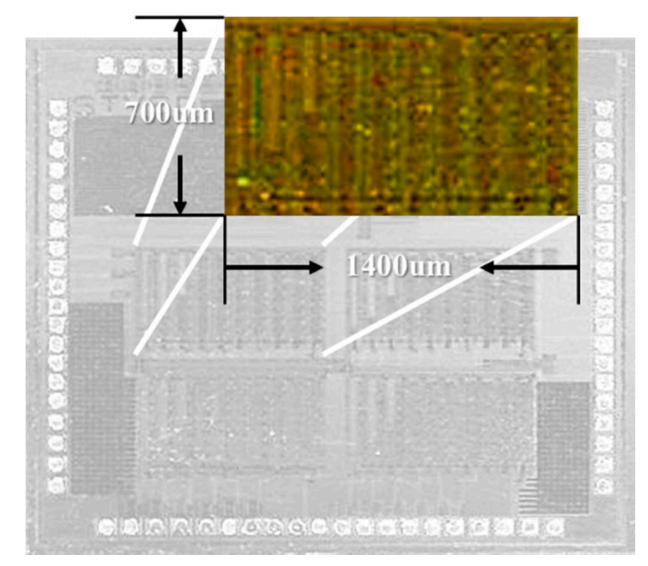

Figure 10 shows the power consumption of the proposed adiabatic design with a constant $\mathrm{VDD}_{\mathrm{DD}}$ The energy dissipation of the conventional operation is observed with $1.13 \mu \mathrm{J}$ in a time period of $150 \mu$ s from the simulation result.

Figure 10. Power consumption of conventional 6-bits LED controller.

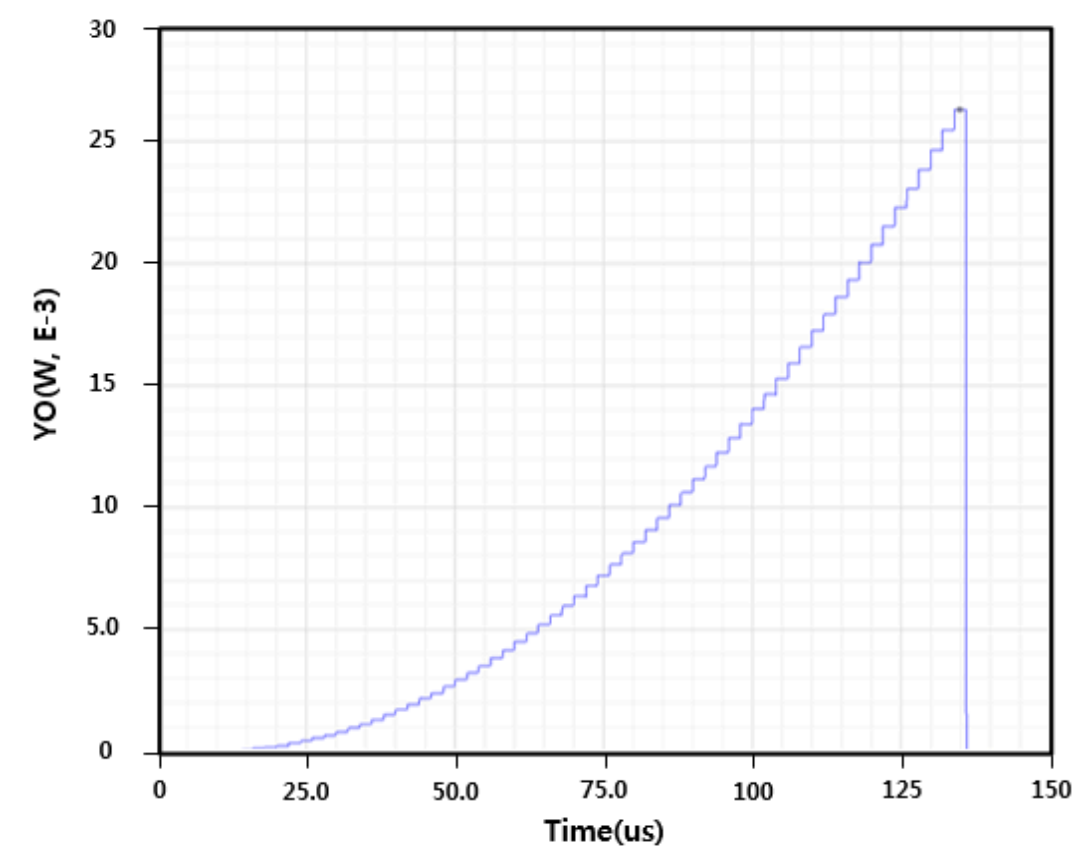

Figure 11 shows the power consumption of the proposed adiabatic design with VTRI. The energy dissipation of adiabatic operation is evaluated with $0.21 \mu \mathrm{J}$ in the period time of $150 \mu \mathrm{s}$. From the simulation result, the power consumption of the proposed LED controller using adiabatic operation was reduced to about $87 \%$ in comparison with the conventional operation with a constant $V_{D D}$. 
Figure 11. Power consumption of adiabatic 6-bits LED controller.

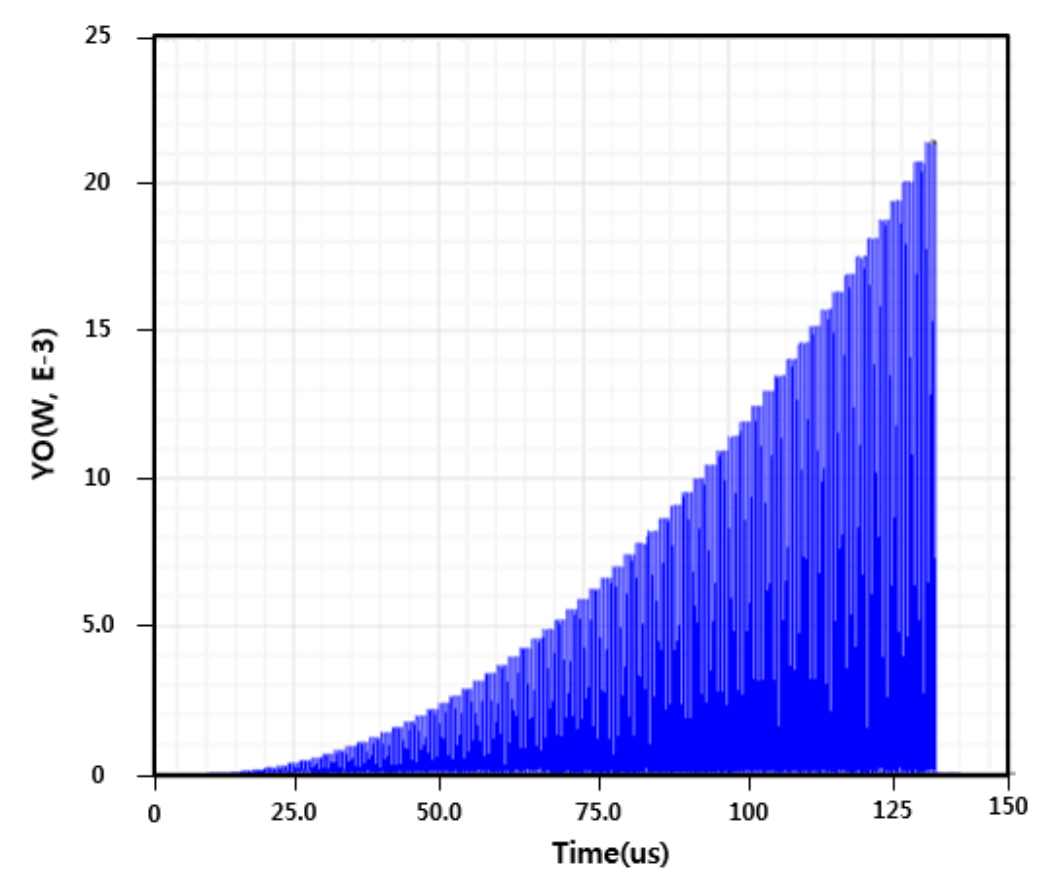

The specific heat of Silicon material as the main component of an LED system is $0.713 \sim 0.8 \mathrm{~J} / \mathrm{g}^{\circ} \mathrm{C}$ [7]. When an LED channel is used in same condition of proposed design, temperature of the LED controller can be limited to $0.26 / 1.41 \mu^{\circ} \mathrm{C}, 18 \%$.

\section{Conclusions}

It is necessary to operate the LED channel increasing, LED controller block with low-power design. In this paper, a low-power technique using adiabatic operation was applied to the LED controller for a stable constant-current, low-power and low-heat function. From the simulation result, the power consumption of the proposed LED controller can be reduced to about $87 \%$ in comparison with conventional operation. The proposed circuit design method is expected to be a solution for the low-power LED controller which is sensitive to external conditions such as heat.

\section{Acknowledgments}

This research was partially funded by the MSIP (Ministry of Science, ICT \& Future Planning), granted. 1391202004, Korea in the ICT R\&D Program 2014.

\section{Author Contributions}

This research was carried out in collaboration among all four authors. Kyung-Ryang Lee defined the research theme. Sung-Dae Yeo designed the circuit with algorithm. Seung-Il Cho carried out the experiments and analyzed the data. Seong-Kweon Kim is the corresponding author. All authors have read and approved the final manuscript. 


\section{Conflicts of Interest}

The authors declare no conflict of interest.

\section{References}

1. Ho, Y.S. Challenging technical issues of 3D video processing. J. Converg. 2013, 4, 1-6.

2. Bae, S.K. Power consumption analysis of prominent time synchronization protocols for wireless sensor networks. J. Inf. Process. Syst. 2014, 10, 300-313.

3. Kwon, D.K.; Chung, K.; Choi, K. A dynamic Zigbee protocol for reducing power consumption. J. Inf. Process. Syst. 2013, 9, 41-52.

4. Dickinson, A.G.; Denker, J.S. Adiabatic dynamic logic. IEEE J. Solid-States Circuit 1995, 30, 311-315.

5. Moon, Y.; Jeong, D.K. An efficient charge recovery logic circuit. IEEE J. Solid-States Circuits 1996, 31, 514-522.

6. Anuar, N.; Takahashi, Y. Adiabatic logic versus CMOS for low power applications. In Proceedings of the 24th International Technical Conference on Circuits/Systems, Computers and Communications (ITC-CSCC), Jeju Island, Korea, 5-8 July 2009; pp. 302-305.

7. Sze, S.M.; Ng, K.K. Physics of Semiconductor Devices, 3rd ed.; Wiley: New York, NY, USA, 2006; p. 790.

(C) 2014 by the authors; licensee MDPI, Basel, Switzerland. This article is an open access article distributed under the terms and conditions of the Creative Commons Attribution license (http://creativecommons.org/licenses/by/4.0/). 